\title{
Identification of microRNA-221/222 and microRNA-323-3p association with rheumatoid arthritis via predictions using the human tumour necrosis factor transgenic mouse model
}

Pandis, Ioannis ; Ospelt, Caroline ; Karagianni, Niki ; Denis, Maria C ; Reczko, Martin ; Camps, Carme ; Hatzigeorgiou, Artemis G ; Ragoussis, Jiannis ; Gay, Steffen ; Kollias, George

\begin{abstract}
OBJECTIVE: To identify novel microRNA (miR) associations in synovial fibroblasts (SF), by performing miR expression profiling on cells isolated from the human tumour necrosis factor (TNF) transgenic mouse model (TghuTNF, Tg197) and patients biopsies.METHODS: miR expression in SF from TghuTNF and wild-type (WT) control mice were determined by miR deep sequencing (miR-seq) and the arthritic profile was established by pairwise comparisons. Quantitative PCR analysis was utilised for profile validation, miR and gene quantitation in patient SF. Dysregulated miR target genes and pathways were predicted via bioinformatic algorithms and validated using gain-of-function coupled with reporter assay experiments.RESULTS: miR-seq demonstrated that TghuTNF-SF exhibit a distinct pathogenic profile with 22 significantly upregulated and 30 significantly downregulated miR. Validation assays confirmed the dysregulation of miR-223, miR-146a and miR-155 previously associated with human rheumatoid arthritis (RA) pathology, as well as that of miR-221/222 and miR-323-3p. Notably, the latter were also found significantly upregulated in patient RA SF, suggesting for the first time their association with RA pathology. Bioinformatic analysis suggested Wnt/cadherin signalling as a putative pathway target. miR-323-3p overexpression was shown to enhance Wnt pathway activation and decrease the levels of its predicted target -transducin repeat containing, an inhibitor of -catenin.CONCLUSIONS: Using miRseq-based profiling in SF from the TghuTNF mouse model and validations in RA patient biopsies, the authors identified miR-221/222 and miR-323-3p as novel dysregulated miR in RA SF. Furthermore, the authors show that miR-323-3p is a positive regulator of WNT/cadherin signalling in RA SF suggesting its potential pathogenic involvement and future use as a therapeutic target in RA.
\end{abstract}

DOI: https://doi.org/10.1136/annrheumdis-2011-200803

Posted at the Zurich Open Repository and Archive, University of Zurich

ZORA URL: https://doi.org/10.5167/uzh-62509

Journal Article

Originally published at:

Pandis, Ioannis; Ospelt, Caroline; Karagianni, Niki; Denis, Maria C; Reczko, Martin; Camps, Carme; Hatzigeorgiou, Artemis G; Ragoussis, Jiannis; Gay, Steffen; Kollias, George (2012). Identification of microRNA-221/222 and microRNA-323-3p association with rheumatoid arthritis via predictions using the human tumour necrosis factor transgenic mouse model. Annals of the Rheumatic Diseases, 71(10):17161723 .

DOI: https://doi.org/10.1136/annrheumdis-2011-200803 


\section{Identification of microRNA-221/222 and -323-3p Association with Rheumatoid Arthritis via Predictions using the Human TNF Transgenic Mouse Model}

\section{Authors:}

loannis Pandis ${ }^{1}$, Caroline Ospelt ${ }^{2,3}$, Niki Karagianni ${ }^{1,4}$, Maria C. Denis ${ }^{4}$, Martin Reczko $^{5}$, Carme Camps ${ }^{6}$, Artemis G Hatzigeorgiou ${ }^{5}$, Jiannis Ragoussis ${ }^{6,7}$, Steffen Gay ${ }^{2,3}$ and George Kollias ${ }^{1, *}$

\section{Author Affliliations:}

${ }^{1}$ Institute of Immunology, Biomedical Sciences Research Center "Alexander Fleming", Vari, Greece

${ }^{2}$ Center of Experimental Rheumatology, University Hospital Zurich and Zurich Center of Integrative Human Physiology, Zurich, Switzerland

${ }^{3}$ Zurich Center of Integrative Human Physiology, Zurich, Switzerland

${ }^{4}$ Biomedcode Hellas SA, Vari, Greece

${ }^{5}$ Institute of Molecular Oncology, Biomedical Sciences Research Center "Alexander Fleming", Vari, Greece

${ }^{6}$ The Wellcome Trust Centre for Human Genetics, University of Oxford, Oxford, UK ${ }^{7}$ Institute of Molecular Biology \& Genetics, Biomedical Sciences Research Center "Alexander Fleming", Vari, Greece

\section{* : Corresponding author}

Full Name: George Kollias, Postal address: Institute of Immunology, Biomedical Sciences Research Center "Alexander Fleming", 16672, Vari, Greece, E-mail: g.kollias@fleming.gr, Telephone: +30-210-965-6507, Fax: +30-210-965-6563 
Keywords: rheumatoid arthritis, microRNA, synovial fibroblast, human TNF transgenic mouse, WNT

Abbreviations used: TghuTNF (Tg197): human TNF transgenic, miR: microRNA, RA: rheumatoid arthritis, OA: osteoarthritis, SF: synovial fibroblast, WT: wild type, PBMC: peripheral blood mononuclear cell, FC: fold change, qPCR: quantitative real time polymerase chain reaction, miR-seq: miRNA deep sequencing, CSNK1A1: casein kinase I isoform alpha, BTRC: beta-transducin repeat containing, GSK3B: glycogen synthase kinase 3 beta. 


\section{ABSTRACT}

OBJECTIVE: To identify novel microRNA (miR) associations in synovial fibroblasts (SFs), by performing miR expression profiling on cells isolated from the human TNF transgenic mouse model (TghuTNF, Tg197) and patients biopsies.

METHODS: miR expression in SFs from TghuTNF and WT control mice were determined by miR deep sequencing (miR-seq) and the arthritic profile was established by pairwise comparisons. qPCR analysis was utilised for profile validation, $\mathrm{miR}$ and gene quantitation in patient SFs. Dysregulated miR target genes and pathways were predicted via bioinformatic algorithms and validated using gain-of-function coupled with reporter assays experiments.

RESULTS: miR-seq demonstrated that TghuTNF-SFs exhibit a distinct pathogenic profile with 22 significantly upregulated and 30 significantly downregulated miRs. Validation assays confirmed the dysregulation of miR-223, miR-146a and miR-155 previously associated with human rheumatoid arthritis (RA) pathology, as well as that of miR221/222 and miR-323-3p. Notably, the latter were also found significantly upregulated in patient RASFs, suggesting for the first time their association with RA pathology. Bioinformatic analysis suggested Wnt/Cadherin signaling as a putative pathway target. miR-323-3p overexpression was shown to enhance Wnt pathway activation and decrease the levels of its predicted target BTRC, an inhibitor of $\beta$-catenin.

CONCLUSIONS: Using miR-seq based profiling in SFs from the TghuTNF mouse model and validations in RA patient biopsies, we identify miR-221/222 and miR-323$3 p$ as novel dysregulated miRs in RASFs. Furthermore, we show that miR-323-3p is a positive regulator of WNT/cadherin signaling in RASFs suggesting its potential pathogenic involvement and future use as a therapeutic target in RA. 


\section{INTRODUCTION}

The multifactorial and complex molecular pathogenesis of RA creates hurdles in the understanding and treatment of this debilitating disease. Animal models of RA have been essential in overcoming patient heterogeneity, thus aiding basic understanding of biological mechanisms, identification and validation of novel pathogenic pathways and evaluation of diagnostic and therapeutic agents.[1] Nevertheless, despite all efforts to date, the aetiology and comprehensive therapy of RA still remains elusive, warranting further study into its molecular mechanisms.

miRs, a class of small non-coding RNA molecules, act as posttranscriptional regulators and are involved in a plethora of cellular functions. They function predominantly by silencing target genes by binding to the $3^{\prime}$ UTR of their mRNA, in a sequence-specific manner, inhibiting mRNA translation or inducing mRNA degradation.[2] miRs are highly abundant and show high stability in biological fluids, with changes in their levels correlating with disease prognosis and/or activity, nominating these molecules valuable disease biomarkers.[3] In addition, miRs have attracted a great deal of attention as potential therapeutic targets, as the sequencespecific mode in which they act allows the simultaneous targeting of multiple target genes, often members of the same biological pathway(s).[4] In the context of RA, miR155, miR-146a, miR-223, miR-16 and miR-132 have been found dysregulated in

patient peripheral blood mononuclear cells (PBMCs) and various biological fluid samples including synovial fluid and plasma, providing a putative diagnostic potential. $[5,6]$ 
A key cell type mediating RA pathogenesis is the SF, with current concepts proposing that therapeutics targeting RASFs could act synergistically with existing approaches leading to more favourable outcomes.[7] Thus, better understanding of the molecular changes occurring in RASFs is paramount for the generation of targeted therapeutics. miR perturbations have been reported in RA patient SFs including the overexpression of miR-155, miR-146a and miR-203, and the underexpression of miR124a.[8-10]

In this study, we hypothesised that additional perturbations in RASF miR expression are currently masked by the heterogeneity present in patient genetic backgrounds and treatment regimes. To overcome this limitation, we used the TghuTNF mouse, a well-established mouse model of human RA that due to huTNF deregulated expression develops spontaneous arthritis characterized by infiltration of inflammatory cells, synovial hyperplasia, cartilage destruction and bone erosion, closely resembling human pathology.[11] The development of full pathology in these mice is not dependent on haematopoietic TNFRI,[12] but interestingly mediated by the direct activation of TNFR1 on SFs.[13] Thus, aiming to identify novel miRs associated with the arthritic phenotype of RASFs we performed miR expression profiling on TghuTNF-SFs and validated the results on human RASFs. 


\section{MATERIALS AND METHODS}

\section{Mice}

TghuTNF mice were generated as previously described.[11] All mice were bred and maintained on a mixed $\mathrm{CBA} \times \mathrm{C} 57 \mathrm{BL} / 6 \mathrm{~J}$ genetic background in the animal facilities of the Biomedical Sciences Research Center (BSRC) Alexander Fleming under specific pathogen-free conditions. All mice were used in accordance with the guidance of the Institutional Animal Care and Use Committee of BSRC Alexander Fleming.

\section{Mouse and human cell isolation and culturing}

Primary mouse SFs were isolated from fully diseased 8-week-old TghuTNF and WT littermate mice (two mice per genotype were used for miR-seq experiment and five to eight mice per genotype were used for profile validation) and cultured for three passages as previously described.[14] Cell purity of all preparations, was accessed via FACS. Representative profiles of the two TghuTNF and two WT SF cultures used for the miR-seq experiment are shown on Figure S1. Human RA and osteoarthritis (OA) SFs were isolated and cultured as previously described.[10] Synovial tissue specimens were obtained during synovectomy or joint replacement surgery from patients with RA and patients with $\mathrm{OA}$, after informed consent was obtained from all patients. The local ethics committee approved the study. All RA patients fulfilled the American College of Rheumatology criteria for the classification of RA.[15] All experiments using human cells were performed with SFs in passages 4-7.

\section{RNA isolation}


Total RNA was isolated from cultured mouse and human cells using the miRVana ${ }^{\mathrm{TM}}$ miR Isolation Kit (Ambion) according to the manufacture's protocol.

\section{miR-seq and miR expression profile generation}

miR-seq

miR-seq was performed on RNA from SFs isolated from two TghuTNF and two WT littermate mice. Libraries were prepared as follows: the 20-30 and 70-100 nucleotide fractions were isolated from 10ug of total RNA after being run in a 15\% Urea-TBE gel (Invitogen) for $1 \mathrm{~h}$. The RNA contained on the excised gel bands was eluted on $300 \mu \mathrm{l}$ of $0.3 \mathrm{M} \mathrm{NaCl}$ solution during $4 \mathrm{~h}$ at $\mathrm{RT}$ and constant rotation. The elute was separated from the gel debris through a Spin-X-column (Fisher) and RNA was precipitated by adding $750 \mu \mathrm{l}$ of $100 \%$ ethanol and $3 \mu \mathrm{l}$ of glycogen (Ambion) $(1 \mathrm{mg} / \mathrm{ml})$ and incubating it for $30 \mathrm{~min}$ at $-80^{\circ} \mathrm{C}$. The precipitated RNA was centrifuged at $14 \mathrm{~K}$ rpm for $25 \mathrm{~min}$ at $4^{\circ} \mathrm{C}$, washed with $75 \%$ ethanol and resuspended on $5.7 \mu \mathrm{l}$ of RNAse free water. A $5^{\prime}$ adaptor was ligated to the RNA in a reaction containing the full amount of RNA recovered from last step, $1.3 \mu$ l of SRA 5 ' adaptor (Illumina), $1 \mu$ I T4 RNA ligase $(10 U / \mu l)$ (Promega), $1 \mu \mathrm{l}$ 10X T4 RNA ligase reaction buffer (Promega) and $1 \mu \mathrm{l}$ RNAseOUT (Invitrogen). The reaction was incubated at $20^{\circ} \mathrm{C}$ in a thermal cycler for $6 \mathrm{~h}$. The resulting product was run on a 15\% Urea-TBE gel (Invitrogen) and the band corresponding to 40-60 nucleotides was excised. The 5' ligated RNA was eluted and precipitated as described before and finally resuspended in $6.4 \mu \mathrm{l}$ of RNAse free water. A $3^{\prime}$ adaptor was added to these molecules in a reaction containing the entire $5^{\prime}$ ligated RNA recovered from last step, $0.6 \mu$ l of SRA 3' adaptor (Illumina), $1 \mu$ I T4 RNA ligase $(10 U / \mu \mathrm{l})$ (Promega), $1 \mu \mathrm{l}$ 10X T4 RNA ligase (Promega) and $1 \mu \mathrm{l}$ RNAseOUT 
(Invitrogen). The reaction was incubated at $20^{\circ} \mathrm{C}$ in a thermal cycler for $6 \mathrm{~h}$. The product obtained was run on a 10\% Urea-TBE gel (Invitrogen) and the band corresponding to 70-90 nucleotides was excised. The 5' and 3' ligated RNA was eluted and precipitated as described before and finally resuspended in $4.5 \mu$ of RNAse free water. In order to synthesize single stranded DNA from this material, $0.5 \mu$ of SRA RTprimer (Illumina) were added to it and the mixture was incubated at $65^{\circ} \mathrm{C}$ for $10 \mathrm{~min}$. This was complemented with $2 \mu$ l of $5 \mathrm{X}$ first strand buffer, $1 \mu \mathrm{l} 100 \mathrm{mM}$ DTT, $0.5 \mu \mathrm{l}$ RNAseOUT (all from Superscript II reverse transcription kit (Invitrogen) and $0.5 \mu \mathrm{l}$ $12.5 \mathrm{mM} \mathrm{dNTP} \operatorname{mix}$ (BioLine) and incubated at $48^{\circ} \mathrm{C}$ for $3 \mathrm{~min}$. After adding $1 \mu \mathrm{l}$ of Superscript II retrotranscriptase (Invitrogen), the reaction was incubated at $44^{\circ} \mathrm{C}$ for $1 \mathrm{~h}$. A PCR reaction was then set up with the resultant product and $0.5 \mathrm{ul}$ GX1 primer, $0.5 \mathrm{ul}$ GX2 primer (both from Illumina), 0.5ul 25mM dNTP mix (Bioline), 10ul 5X cloned Phu buffer, 0.5ul Phu polymerase (both from NEB) and 28ul RNAse free water. The cycling conditions were as follows: 1 cycle of $98^{\circ} \mathrm{C}$ for $30 \mathrm{sec} ; 15$ cycles of $98^{\circ} \mathrm{C}$ for $10 \mathrm{sec}$, $60^{\circ} \mathrm{C}$ for $30 \mathrm{sec}$ and $72^{\circ} \mathrm{C}$ for $15 \mathrm{sec} ; 1 \mathrm{cycle}$ of $72^{\circ} \mathrm{C}$ for $10 \mathrm{~min}$. The PCR product was purified by running it on a 10\% TBE-PAGE gel for $35 \mathrm{~min}$. The band around 90bp was excised and eluted on 100ul of $1 \mathrm{X}$ Elution buffer 2 (NEB) for $2 \mathrm{~h}$ at RT and at constant rotation. The elute was separated from the gel debris through a Spin-X-column (Fisher) and DNA was precipitated by adding $1 \mu$ l of glycogen $(1 \mathrm{mg} / \mathrm{ml})$ (Ambion), $10 \mathrm{ul} 3 \mathrm{M}$ sodium acetate (Ambion) and $325 \mu$ of $-20^{\circ} \mathrm{C} 100 \%$ ethanol and centrifuging at $14 \mathrm{~K}$ rpm for 20 min. After a wash with $70 \%$ ethanol, the DNA was vacuum dried and resuspended in 10 ul of water. The libraries were sequenced using an Illumina GA II machine according to manufacturer's standard protocols at 36bp read length. 


\section{Alignment algorithm}

Our short sequence alignment method is based on a de Bruijn graph representation of the reference collection of known miR sequences.[16] This collection contained all murine major and minor (or star) mature miRNAs as defined in mirBase release 15.0, giving a total of 707 sequences. All 9mers occurring in each reference sequence and its reverse complement were stored in a hash table together with an identifier for the reference sequence and its start position within the sequence. For each read, all valid paths through the reference sequences were collected, allowing for up to 2 sequence edits (mismatches, insertions or deletions).

TghuTNF-SF miR expression profile generation

For miR expression profile generation miR transcript abundance (sequencing reads) was used as a measure of expression. To allow pairwise comparisons, as a common practice, miR read numbers in each sample were normalised to the total reads per million. All miRs that had less than 6 reads in any of the samples were considered as not being expressed and discarded. Subsequently, expression fold change (FC) was calculated by dividing the average TghuTNF reads by the average WT reads. miRs showing a FC of less than 1.5 were also discarded. Significant differences in the expression of the remaining 141 miRs between the two groups were determined using an unpaired two-sided t-test, on the log2-transformed reads, as proposed by Creighton et al.[17] miRs with a p-value of less than 0.05 , fold change greater than 1.5 and a false discovery rate (FDR) of less than 5\% (calculated according to Storey JD and Tibshirani R.,2003)[18] were considered significantly dysregulated. All Sequencing Data has been deposited on Gene Expression Omnibus (GEO) with ID: GSE31667. 


\section{Profile validation and human patient $\mathrm{SF}$ miR and gene expression quantitation}

Profile validation was performed on TghuTNF ( $n \geq 5)$ and WT $(n \geq 5)$ SFs isolated in independent experiments, using quantitative real-time polymerase chain reaction (qPCR) based TaqMan ${ }^{\circledR}$ MiR Assays (Applied Biosystems) according to the manufacturer's instructions, on a Chromo4 Real-Time PCR detection system (Bio-Rad Laboratories). Small nuclear RNA U6 (snU6) was used as an endogenous control for normalisation purposes and expression FCs were calculated using the $\Delta \Delta \mathrm{C}(\mathrm{t})$ method. [19] Samples with a $C(t)>35$ were considered below detection.

Human patient quantitations of miRs, CSNK1A1, BTRC and GSK3B were performed on RASFs and OASFs as a control, using the same qRT-PCR assays described above, on a 7500 real-time PCR system (Applied Biosystems). let-7a miR was used as an endogenous control for miR measurements while ribosomal $18 \mathrm{~S}$ levels were used for mRNA measurements. Differences in expression were assessed using the $\Delta \mathrm{C}(\mathrm{t})$ method, as previously described.[9,10] For the detection of CSNK1A1, BTRC and GSK3B, SYBR ${ }^{\circledR}$ dye and the following primers pairs were used: CSNK1A1 forward (fw) CGGCGAGGAAGTGGCAGTGA, reverse (rev) TGGGGATGCCTGGCCTTCTGA; BTRC fw CCCGTGCTCCTGCAGGGACA, rev CGGAATGCTCCACAAGGGTCCG; GSK3B

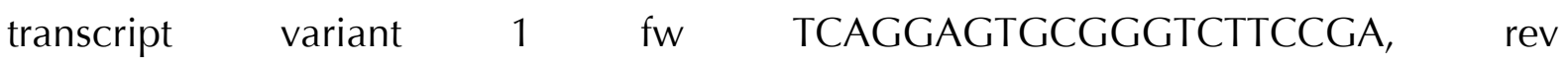

CAGTGCAATTGCCTCCGGTGGA; GSK3B transcript variant $2 \mathrm{fw}$ TTGGACTAAGGTCTTCCGACCCCG, rev CAGTGCAATTGCCTCCGGTGGA. 


\section{Bioinformatics}

miR expression clustering and heatmap generation

Pearson correlation based clustering and heatmap generation of miR expression profiles was calculated using the made4 package for the the $R$ statistics environment.[20]

miR target prediction and pathway analysis

Human and mouse miR target predictions were performed using the DIANA microT and TargetScan algorithms.[21,22] The g:Profiler tool was used for all gene ID conversion purposes [23] and the Panther repository was used for pathway enrichment analysis.[24]

For each species miR targets, we combined the unions of the two algorithms outputs, which weight species conservation of predicted targets differentially, producing more sensitive target lists. By comparing the mouse and human gene target lists we generated for each miR lists of conserved, mouse specific and human specific targets (Supplementary excel). Pathway enrichment analysis was performed on the conserved lists, using the species specific lists as controls to exclude random enrichment, against the default Homo sapiens reference gene list provided by Panther. Pathways with pvalue $<0.001$ were considered significantly enriched.

\section{miR overexpression and WNT reporter assays}

Co-transfections of a human cell line with a WNT reporter plasmid and miR precursors (pre-miRs) were performed to clarify $\mathrm{miR}$ and pathway relationship, whereas, transfections of RASFs with pre-miRs were used to assess the regulatory potential of 
miRs on target genes. HEK 293T cells were used for reporter plasmid transfections (a kind gift from Dr Hatzis, Fleming, Athens). Pre-miRTM miRNA Precursors (Ambion) were used for miR overexpression. The M50 Super 8x TOPFlash[25] wnt repoter plasmid was purchased from Addgene and a CMV/GFP expressing plasmid was used to control transfection efficiency. The Lipofectamine® 2000 (Invitrogen) reagent was used for plasmid and pre-miR transfections and the Steady-Gloß Luciferase Assay System was used for luciferase activity quantitation. Luminescence and GFP fluorescence were measured on an Infinite ${ }^{\circledR}$ M200 multimode reader (Tecan). Assays were performed according to the Pre-miR ${ }^{T M}$ miRNA Precursor guidelines.

Briefly, for each transfection $10^{4}$ HEK 293T cells were seeded in one well of Corning ${ }^{\circledR}$ 96 Well Flat Clear Bottom White Polystyrene TC plates and transfected using the lipidmediated forward co-transfection method with $50 \mathrm{nM}$ pre-miR $(25 \mathrm{nM}$ of each miR in the case of miR-221/222), 40ng Super 8x TOPFlash plasmid and 4ng CMV/GFP for 48 hours. After 48 hours, the WNT pathway was activated by treating the cells with $10 \mathrm{mM}$ $\mathrm{LiCl}$ for 16 hours, followed by cell lysis and quantitation of luminescence and fluorescence. Each miR, including the control, was transfected four times in each experiment and each experiment was repeated three times.

For transfection of RASFs with pre-miRs, cells were seeded at $50 \times 10^{4} /$ well in 12 well plates and transfected with $50 \mathrm{nM}$ pre-miR $\mathrm{R}^{\mathrm{TM}} 323-3 \mathrm{p}$ or pre-miR $\mathrm{R}^{\mathrm{TM}}$ negative control (Ambion) using Lipofectamine ${ }^{\circledR} 2000$ (Invitrogen). Medium was changed after 24h,

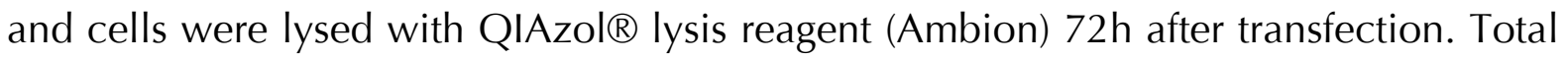
RNA was isolated using the miRVana ${ }^{\mathrm{TM}}$ miR Isolation Kit (Ambion) and expression of target genes was measured with the 7500 real-time PCR system as described above. 


\section{Statistics}

Statistical analyses were performed using GraphPad Prism. For profile validation purposes, significant $(p<0.05)$ FCs between the two groups were computed using a one-sample t-test. Pearson's correlation coefficient was used as similarity measure of miR-seq and qPCR based FC, after Gaussian distribution was checked using the Shapiro-Wilk's test.

Significant differences $(\mathrm{p}<0.05)$ between RA and OA SFs miR expression were assessed using unpaired two-tailed t-tests on qPCR $\triangle \mathrm{C}(\mathrm{t})$ values, after Gaussian distribution was checked using the Kolmogorov-Smirnov test for $n=5$ or Shapiro-Wilk's for $n \geq 6$. 


\section{RESULTS}

\section{TghuTNF-SFs exhibit a distinct pathogenic miR expression profile overlapping with that of RA patient SFs}

The miR expression profiles of SFs isolated from two fully diseased 8week old TghuTNF and two healthy WT littermate mice were determined by miR-seq. Pairwise significance analysis of the two profiles showed that $22 \mathrm{miRs}$ are upregulated and 30 miRs downregulated in TghuTNF-SFs, FC $>1.5$, p-value $<0.05$ and $\mathrm{FDR}<5 \%$ (Figure $1 \mathrm{~A}$ and Table S1). All Sequencing Data has been deposited on GEO with ID: GSE31667. Profile validation was performed on a panel of 13 randomly selected miRs via qPCR, on independently isolated pairs of SFs $(n \geq 5)$. The upregulation of miR-223, miR-146a, miR-155, miR-221, miR-222 and miR-323-3p, and downregulation of miR-322 and miR-335 in TghuTNF-SFs was confirmed, while the dysregulation of miR-138 and miR503 was not and miR-122a, miR-203 and miR-378 levels were below the assay detection capacity (Figure 1C and Table S1).

The validity and quantitative capacity of the sequence-based expression profile was further underlined by the significant positive correlation $(r=0.9782, p<0.0001$, $\mathrm{n}=10$ ) of miR-seq and qPCR based FC, determined via Pearson correlation analysis (Figure 1D).

Current literature on the association of miRs with RASFs report miR-155, miR146a and miR-203 overexpression, and reduced levels of miR-124a in RASFs compared to OASFs.[8-10] Our results show that miR-203 is downregulated in the TghuTNF-SF profile, but was below detection in qPCR validation assays (Figure 1A, 1C, 
Table S1 and S2). In addition, miR-124 transcripts could not be detected in any of the four samples sequenced (Data not shown). In contrast, miR-155 and miR-146a are indeed over-expressed in TghuTNF-SFs (Figure 1A, 1C and Table S2), indicating that the profile overlaps with the current RA patient SF findings.

\section{TghuTNF-SF and RASF miR expression profile comparison identifies miR-221/222 and miR-323-3p association with RASFs}

Next we examined our hypothesis that additional miR perurbations are shared between TghuTNF-SFs and RASFs. To this end, the validated mouse profile miRs were quantitated via qPCR in patient RASFs $(n=8)$ using OASFs $(n=8)$ as a control.

No significant differences were found in miR-223, miR-335, miR-424 (homologue of mouse miR-322) or notably miR-146a expression, which has previously been reported to be altered in RASFs.[10] This incomplete penetrance of miR-146a may be due to heterogeneity present in patient samples. Interestingly, miR-155 dysregulation was verified and most notably we found that miR-221, miR-222 and miR-323-3p are significantly over-expressed in RASFs, establishing for the first time their association with RA (Figure 2, Table S3).

\section{In silico predictions of miR-221/222 and miR-323-3p conserved targets and pathways}

Subsequently, we investigated the putative functional role of the newly associated miRs. To this end, pathway enrichment analysis was performed using Panther [24] on sensitive lists (Materials and Methods, Supplementary excel) of conserved miR gene 
targets in humans and mice, predicted by two major target prediction algorithms, namely DIANA microT and TargetScan.[21,22] Human and mouse specific target lists (Supplementary excel) were used as controls, to exclude enrichment due to redundancy often observed in these types of analyses.

miR-221 and miR-222 were studied simultaneously, as they are a co-expressed cluster of miRs, possessing identical 5' seed regions targeting the same genes. The miR221/222 conserved target gene list (978 genes) showed a significant enrichment $(p<0.001)$ in 11 pathways, whereas 3 pathways were enriched in the mouse specific target list (1420 genes) and the human specific list (2040 genes) showed no significant enrichment in any pathways (Table 2 and Supplementary excel). The miR-323-3p analysis revealed a significant enrichment in 18 pathways in the conserved target list (1711 genes), no significant enrichment in mouse specific list (1326 genes) and one in the human specific list (3077 genes) (Table 3 and Supplementary excel). All target genes involved in enriched pathways are provided (Supplementary excel).

Interestingly, pathways previously associated with RA pathogenesis, such as wnt,[26,27] cadherin, [28] angiogenesis, [29] Ras,[30] PI3K,[31] PDGF,[32] T cell activation[33] and integrin[29] pathways were enriched in both miR target lists, implying that the newly identified miRs may modulate more than one key arthritogenic pathways.

Table 2. Pathway enrichment analysis of miR-221/222 conserved predicted target genes. (ONLINE ONLY MATERIAL)

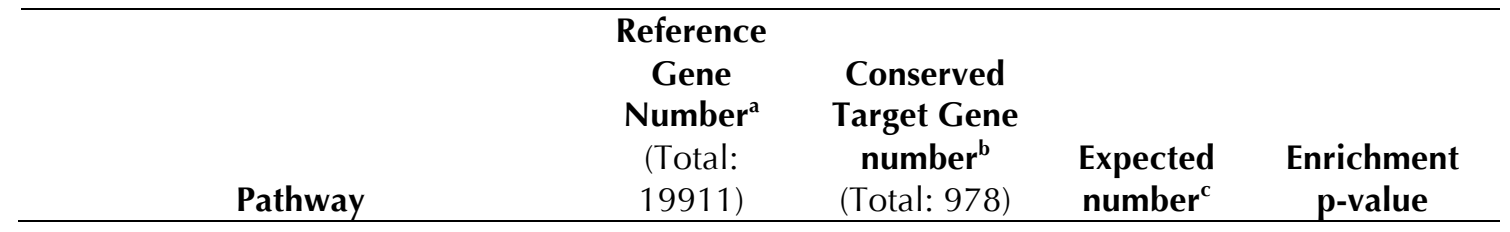




\begin{tabular}{ccccc}
\hline Wnt signaling pathway & 317 & $\mathbf{4 6}$ & 15.57 & $\mathbf{2 . 0 3 E - 1 0}$ \\
Cadherin signaling pathway & 147 & $\mathbf{2 6}$ & 7.22 & $\mathbf{4 . 3 4 E - 0 8}$ \\
Angiogenesis & 191 & $\mathbf{2 7}$ & 9.38 & $\mathbf{1 . 7 6 E - 0 6}$ \\
Alzheimer disease-presenilin & 122 & $\mathbf{1 8}$ & 5.99 & $\mathbf{5 . 2 2 E - 0 5}$ \\
$\begin{array}{c}\text { pathway } \\
\text { Ras Pathway }\end{array}$ & 79 & $\mathbf{1 4}$ & 3.88 & $\mathbf{5 . 3 0 E - 0 5}$ \\
$\begin{array}{c}\text { PI3 kinase pathway } \\
\text { Metabotropic glutamate receptor }\end{array}$ & 115 & $\mathbf{1 6}$ & 5.65 & $\mathbf{2 . 5 4 E - 0 4}$ \\
$\begin{array}{c}\text { group Il pathway } \\
\text { Muscarinic acetylcholine receptor }\end{array}$ & 51 & $\mathbf{1 0}$ & 2.51 & $\mathbf{2 . 7 5 E - 0 4}$ \\
2 and 4 signaling pathway & 62 & $\mathbf{1 1}$ & 3.05 & $\mathbf{3 . 2 1 E - 0 4}$ \\
$\begin{array}{c}\text { Metabotropic glutamate receptor } \\
\text { group III pathway }\end{array}$ & 73 & $\mathbf{1 2}$ & 3.59 & $\mathbf{3 . 4 6 E - 0 4}$ \\
$\begin{array}{c}\text { PDGF signaling pathway* } \\
\text { T cell activation }\end{array}$ & 159 & $\mathbf{1 9}$ & 7.81 & $\mathbf{4 . 6 1 E - 0 4}$ \\
\hline
\end{tabular}

${ }^{a}$ Number of human genes in panther pathway

${ }^{b} N u m b e r$ of conserved targets genes in panther pathway

'Expected number of conserved target genes based on list size

*Also enriched in mouse specific target gene list ( $p$-value=2.90e-04) (supplementary excel file)

Table 3. Pathway enrichment analysis of miR-323-3p conserved predicted target genes. (ONLINE ONLY MATERIAL)

\begin{tabular}{|c|c|c|c|c|}
\hline Pathway & $\begin{array}{c}\text { Reference Gene } \\
\text { Number }^{\mathrm{a}} \\
\text { (Total: 19911) }\end{array}$ & $\begin{array}{c}\text { Conserved } \\
\text { Target Gene } \\
\text { number }^{b} \\
\text { (Total: } 1711 \text { ) } \\
\end{array}$ & $\begin{array}{l}\text { Expected } \\
\text { number }^{c}\end{array}$ & $\begin{array}{c}\text { Enrichment } \\
\text { p-value }\end{array}$ \\
\hline Wnt signaling pathway & 317 & 69 & 27.24 & 8.98E-12 \\
\hline $\begin{array}{c}\text { Cadherin signaling } \\
\text { pathway }\end{array}$ & 147 & 35 & 12.63 & $1.49 \mathrm{E}-07$ \\
\hline Angiogenesis & 191 & 37 & 16.41 & 7.61E-06 \\
\hline $\begin{array}{c}\text { Inflammation mediated } \\
\text { by chemokine and } \\
\text { cytokine signaling } \\
\text { pathway }\end{array}$ & 283 & 46 & 24.32 & 4.99E-05 \\
\hline $\begin{array}{l}\text { Metabotropic } \\
\text { glutamate receptor } \\
\text { group II pathway }\end{array}$ & 51 & 15 & 4.38 & $5.35 \mathrm{E}-05$ \\
\hline $\begin{array}{c}\text { Endothelin signaling } \\
\text { pathway }\end{array}$ & 91 & 21 & 7.82 & 6.56E-05 \\
\hline $\begin{array}{l}\text { EGF receptor signaling } \\
\text { pathway }\end{array}$ & 135 & 27 & 11.6 & 7.28E-05 \\
\hline $\begin{array}{l}\text { Metabotropic } \\
\text { glutamate receptor } \\
\text { group III pathway }\end{array}$ & 73 & 18 & 6.27 & $9.47 \mathrm{E}-05$ \\
\hline $\begin{array}{l}\text { Axon guidance } \\
\text { mediated by } \\
\text { semaphorins }\end{array}$ & 43 & 13 & 3.7 & $1.26 \mathrm{E}-04$ \\
\hline $\begin{array}{l}\text { Integrin signalling } \\
\text { pathway }\end{array}$ & 181 & 32 & 15.55 & $1.58 \mathrm{E}-04$ \\
\hline $\begin{array}{l}\text { PDGF signaling } \\
\text { pathway }\end{array}$ & 159 & 29 & 13.66 & 1.89E-04 \\
\hline Ras Pathway & 79 & 18 & 6.79 & $2.44 \mathrm{E}-04$ \\
\hline
\end{tabular}




\begin{tabular}{|c|c|c|c|c|}
\hline $\mathrm{T}$ cell activation & 102 & 21 & 8.77 & 3.01E-04 \\
\hline $\begin{array}{c}\text { Heterotrimeric G- } \\
\text { protein signaling } \\
\text { pathway-Gq alpha and } \\
\text { Go alpha mediated } \\
\text { pathway }\end{array}$ & 134 & 25 & 11.51 & 3.65E-04 \\
\hline $\begin{array}{l}\text { Ubiquitin proteasome } \\
\text { pathway }\end{array}$ & 70 & 16 & 6.02 & 5.09E-04 \\
\hline Parkinson disease & 100 & 20 & 8.59 & $5.88 \mathrm{E}-04$ \\
\hline $\begin{array}{l}\text { Apoptosis signaling } \\
\text { pathway }\end{array}$ & 123 & 23 & 10.57 & 5.95E-04 \\
\hline $\begin{array}{l}\text { GABA- } \\
\text { B_receptor_II_signaling }\end{array}$ & 40 & 11 & 3.44 & 8.70E-04 \\
\hline
\end{tabular}

\section{miR-323-3p enhances Wnt/cadherin pathway activation}

Pathway enrichment analysis indicated Wnt and Cadherin signaling as the top pathways potentially affected by both miR-221/222 and miR-323-3p overexpression. Activation of wnt/cadherin signaling in RA has been reported and the constitutive upregulation of $\beta$-catenin, a common component of both pathways, [34] has been linked to the RASFs activated phenotype.[26-28,35,36] Accordingly, gain of function experiments showed that WNT reporter activity induced by lithium salt treatment,[37] was significantly increased in HEK 293T cells overexpressing miR-323-3p (Figure 3A). miR-221/222 overexpression showed no effect.

Interestingly, CSNK1A1 (casein kinase I isoform alpha), BTRC (beta-transducin repeat containing) and GSK3B (glycogen synthase kinase 3 beta) that are known mediators of the degradation of $\beta$-catenin, [38] were predicted targets of miR-323-3p (Supplementary excel). Subsequent qPCR determination of their expression levels showed that while CSNK1A1 expression was not significantly changed, both GSK3B 
transcript variants, GSK3Btr1 and GSK3Btr2, and BTRC expression was found significantly decreased in RA compared to OA SFs (Figure 3B).

To determine if BTRC and/or GSK3B are regulated by miR-323-3p, we overexpressed miR-323-3p in RASFs, which led to the significant decrease of BTRC mRNA, whereas neither of the GSK3B transcripts was affected (Figure 3C). In addition, miR-323-3p and BTRC expression levels showed a significant inverse correlation in RASFs ( $r=-0.5336, p=0.037$ ) (Figure 3D). Collectively these results suggest that miR323-3p overexpression leads to enhanced activation of $\mathrm{Wnt} /$ cadherin pathways, in part by targeting BTRC in RASFs. 


\section{DISCUSSION}

miRs have been proposed as potentially valuable targets in RA with current literature reporting the dysregulated expression of miR-223, miR-155, miR-146a and miR-16 in patient PBMCs and synovial fluid, reduced miR-132 levels in RA plasma and miR-155, miR-146a, miR-203 and miR-124a dysregulations in patient SFs.[5,6,8-10] miR functional studies suggest a protective role for miR-155 in RASFs, as it has been shown to reduce matrix metalloproteinase (MMP)-1 and -3 expression.[10] Conversely, in vivo functional experiments using miR-155 deficient mice have indicated a pathogenic role for miR-155 in immune cells, since deficient mice are protected against antigen driven arthritis, through the blockade of antigen-specific T-helper 17 (Th17) polarization and show reduced immune-mediated bone destruction in the serum-transfer model of arthritis by the partial inhibition of osteoclastogenesis.[39,40] Furthermore, despite miR-146a upregulation in various RA patient cell types suggesting a pathogenic role, in vivo exogenous double stranded miR-146a administration after the induction of autoantigen driven arthritis in mice, led to a decrease in bone erosion via the partial inhibition of osteoclastogenesis, with no effect on joint inflammation.[41]

Whereas studies on human patient samples are paramount in the direct identification of disease determinants and therapeutic targets, their heterogeneity owing to diverse genetic backgrounds, environmental conditions and treatment regimes impedes this discovery process. Animal models of disease, such as the TghuTNF model, although not fully representing the heterogeneity seen in RA, they do show pathogenic and therapeutic alignment with aspects of human disease, providing invaluable tools to overcome such hurdles due to their homogeneity and complete 
disease penetrance. Thus, comparative studies utilising both sample types should be advantageous in the identification of novel pathogenic mechanisms or biomarkers. [1]

In this study, we overcame limitations possessed by patient heterogeneity by using the TghuTNF mouse. We focused on SFs, which are key cells driving human RA pathology and can alone instigate full pathology in this mouse model.[12,13] By comparing the mouse and human arthritic profiles we found that they overlap and importantly we established a miR-221/222 and miR-323-3p association with RASFs. The TghuTNF-SF miR expression profile is provided here as a resource, which may contain further currently unidentified miR dysregulations associated with human pathology.

A search of current literature did not identify any evidence to support a function for miR-323-3p, in contrast miR-221/222 is an established "oncomiR" cluster overexpressed in various human cancers. The cluster mediates oncogenic transformation in cancer cells by enhancing cellular proliferation, inhibiting apoptosis and enhancing cellular migration.[42] These functions are also altered in RASFs, [43,44] suggesting that they may be regulated by miR-221/222 overexpression.

Furthermore, utilising in silico target predictions and pathway enrichment analysis, we investigated putative target genes and pathways modified by the newly associated miRs. Interestingly, the most significantly enriched pathways were Wnt and Cadherin signaling. The importance of the Cadherin signaling in RA has been established, as Cadherin-11 is necessary for the mounting of an inflammatory response by RASFs and maintenance of the correct architecture of the synovium. $[28,36]$ In addition, the role of the Wnt pathway has also been initially highlighted by a study by Diarra et al, as the inhibition of its key regulator Dickkopf-1 (DKK-1) results in the reversal of the bone- 
destructive phenotype of TghuTNF mice and DKK-1 was shown to be highly expressed in RASFs.[26] Moreover, a recent study has further underscored this association of the WNT pathway in RASFs, by showing that the pathway activation is epigenetically controlled by the enhancer of zeste homologue 2 (EZH2).[27] These key pathways contributing to the pathogenic phenotype of RASFs converge at $\beta$-catenin.[34]

Notably, we show here that miR-323-3p enhances Wnt activation. In addition, miR-323-3p overexpression, led to the decreased levels of the negative regulator of $\beta$ catenin, BTRC, implying that cadherin signaling may also be modulated. We propose that BTRC is only one of the perturbed Wnt/cadherin signaling components and that additional key molecules are also regulated by miR-323-3p and/or other miRs dysregulated in RASFs. Jointly, these results propose the dysregulation of the Wnt/Cadherin pathway as a potential pathogenic target of miR-323-3p in RASFs and that intervention using miR-323-3p inhibitors may be of therapeutic value. 


\section{Contributorship Statement}

IP performed experiments, analysed data and wrote the manuscript. CO performed experiments and analysed data. NK and MD designed and performed experiments and wrote the manuscript. MR designed and analysed data. CC performed experiments. $\mathrm{AH}, \mathrm{JR}$ and SG designed experiments and analysed data. GK designed experiments, analysed data, wrote the manuscript. All authors revised the manuscript. IP, CO, MR and GK are guarantors.

\section{Acknowledgements}

The authors would like to thank Dr Fotsis and Dr Christoforidis for discussions, Dr Moon for constructing the Super 8x TOPFlash plasmid, Dr Hatzis for providing cell lines and together with Dr Bertrand and Dr Dell'accio providing invaluable technical advice. Finally, IP would like to dedicate the paper to the late Mrs. M. Batten for continuous moral support.

\section{Funding}

This project was funded by the Masterswitch Project (HEALTH-F2-2008-223404), EURO-RA RTN (HPRN-CT-2002-00255) and IMI BtCure (grant agreement No 115142) grants to GK and SG. JR was supported by the Wellcome Trust grant 075491/Z/04. SG also received funding from IAR-EPALINGES. 


\section{Licence for Publication}

The Corresponding Author has the right to grant on behalf of all authors and does grant on behalf of all authors, an exclusive licence (or non exclusive for government employees) on a worldwide basis to the BMJ Publishing Group Ltd to permit this article to be published in ARD and any other BMJPGL products and sublicences such use and exploit all subsidiary rights.

Competing Interest: None declared. 


\section{References}

1 Kollias G, Papadaki P, Apparailly F, et al. Animal models for arthritis: innovative tools for prevention and treatment. Ann Rheum Dis. 2011;70:1357-62.

2 Bartel DP. MicroRNAs: genomics, biogenesis, mechanism, and function. Cell 2004;116:281-97.

3 Wang Y, Li Z, He C, et al. MicroRNAs expression signatures are associated with lineage and survival in acute leukemias. Blood Cells Mol Dis. 2010;44:191-7.

4 Montgomery RL, van Rooij E. MicroRNA regulation as a therapeutic strategy for cardiovascular disease. Curr Drug Targets. 2010;11:936-42.

5 Murata K, Yoshitomi H, Tanida S, et al. Plasma and synovial fluid microRNAs as potential biomarkers of rheumatoid arthritis and osteoarthritis. Arthritis Res Ther. 2010;12:R86.

6 Pauley $\mathrm{KM}$, Satoh $\mathrm{M}$, Chan $\mathrm{AL}$, et al. Upregulated miR-146a expression in peripheral blood mononuclear cells from rheumatoid arthritis patients. Arthritis Res Ther. 2008;10:R101.

7 Bartok B, Firestein GS. Fibroblast-like synoviocytes: key effector cells in rheumatoid arthritis. Immunol Rev. 2010;233:233-55.

8 Kawano S, Nakamachi Y. miR-124a as a key regulator of proliferation and MCP1 secretion in synoviocytes from patients with rheumatoid arthritis. Ann Rheum Dis. 2011;70 Suppl 1:i88-91.

9 Stanczyk J, Ospelt C, Karouzakis E, et al. Altered expression of miR-203 in rheumatoid arthritis synovial fibroblasts and its role in fibroblast activation. Arthritis Rheum. 2010;63:373-81. 
10 Stanczyk J, Pedrioli DML, Brentano F, et al. Altered expression of MicroRNA in synovial fibroblasts and synovial tissue in rheumatoid arthritis. Arthritis Rheum. 2008;58:1001-9.

11 Keffer J, Probert L, Cazlaris $\mathrm{H}$, et al. Transgenic mice expressing human tumour necrosis factor: a predictive genetic model of arthritis. EMBO J. 1991;10:402531.

12 Blüml S, Binder NB, Niederreiter B, et al. Antiinflammatory effects of tumor necrosis factor on hematopoietic cells in a murine model of erosive arthritis. Arthritis Rheum. 2010;62:1608-19.

13 Armaka M, Apostolaki M, Jacques $\mathrm{P}$, et al. Mesenchymal cell targeting by TNF as a common pathogenic principle in chronic inflammatory joint and intestinal diseases. J Exp Med 2008;205:331-7.

14 Armaka M, Gkretsi V, Kontoyiannis D, et al. A standardized protocol for the isolation and culture of normal and arthritogenic murine synovial fibroblasts. Protocol Exchange 2009. doi:10.1038/nprot.2009.102

15 Arnett FC, Edworthy SM, Bloch DA, et al. The American Rheumatism Association 1987 revised criteria for the classification of rheumatoid arthritis. Arthritis Rheum. 1988;31:315-24.

16 Pevzner PA, Tang $\mathrm{H}$, Waterman MS. An Eulerian path approach to DNA fragment assembly. Proc Natl Acad Sci U S A. 2001;98:9748-53.

17 Creighton CJ, Reid JG, Gunaratne PH. Expression profiling of microRNAs by deep sequencing. Brief Bioinform. 2009;10:490-7.

18 Storey JD, Tibshirani R. Statistical significance for genomewide studies. Proc Natl Acad Sci U S A. 2003;100:9440-5. 
19 Livak KJ, Schmittgen TD. Analysis of relative gene expression data using realtime quantitative PCR and the 2(-Delta Delta C(T)) Method. Methods 2001;25:402-8.

20 Culhane AC, Thioulouse J, Perrière G, et al. MADE4: an R package for multivariate analysis of gene expression data. Bioinformatics 2005;21:2789-90.

21 Friedman $\mathrm{RC}$, Farh $\mathrm{KK}-\mathrm{H}$, Burge $\mathrm{CB}$, et al. Most mammalian mRNAs are conserved targets of microRNAs. Genome Res. 2009;19:92-105.

22 Maragkakis M, Vergoulis T, Alexiou P, et al. DIANA-microT Web server upgrade supports Fly and Worm miRNA target prediction and bibliographic miRNA to disease association. Nucleic Acids Res. 2011;39:W145-8.

23 Reimand J, Kull M, Peterson $\mathrm{H}$, et al. g:Profiler--a web-based toolset for functional profiling of gene lists from large-scale experiments. Nucleic Acids Res. 2007;35:W193-200.

24 Thomas PD, Kejariwal A, Guo N, et al. Applications for protein sequencefunction evolution data: mRNA/protein expression analysis and coding SNP scoring tools. Nucleic Acids Res. 2006;34:W645-50.

25 Veeman MT, Slusarski DC, Kaykas A, et al. Zebrafish prickle, a modulator of noncanonical Wnt/Fz signaling, regulates gastrulation movements. Curr Biol. 2003;13:680-5.

26 Diarra D, Stolina M, Polzer K, et al. Dickkopf-1 is a master regulator of joint remodeling. Nat Med 2007;13:156-63.

27 Trenkmann $M$, Brock $M$, Gay RE, et al. Expression and function of EZH2 in synovial fibroblasts: epigenetic repression of the Wnt inhibitor SFRP1 in rheumatoid arthritis. Ann Rheum Dis. 2011;70:1482-8.

28 Lee DM, Kiener HP, Agarwal SK, et al. Cadherin-11 in synovial lining formation and pathology in arthritis. Science 2007;315:1006-10. 
29 Koch AE. Review: angiogenesis: implications for rheumatoid arthritis. Arthritis Rheum. 1998;41:951-62.

30 Trabandt A, Aicher WK, Gay RE, et al. Expression of the collagenolytic and Rasinduced cysteine proteinase cathepsin $\mathrm{L}$ and proliferation-associated oncogenes in synovial cells of MRL/I mice and patients with rheumatoid arthritis. Matrix. 1990;10:349-61.

31 Kim K-W, Cho M-L, Park M-K, et al. Increased interleukin-17 production via a phosphoinositide 3-kinase/Akt and nuclear factor kappaB-dependent pathway in patients with rheumatoid arthritis. Arthritis Res Ther. 2005;7:R139-48.

32 Rosengren S, Corr M, Boyle DL. Platelet-derived growth factor and transforming growth factor beta synergistically potentiate inflammatory mediator synthesis by fibroblast-like synoviocytes. Arthritis Res Ther. 2010;12:R65.

33 Burger D, Rezzonico R, Li JM, et al. Imbalance between interstitial collagenase and tissue inhibitor of metalloproteinases 1 in synoviocytes and fibroblasts upon direct contact with stimulated $\mathrm{T}$ lymphocytes: involvement of membraneassociated cytokines. Arthritis Rheum. 1998;41:1748-59.

34 Nelson WJ, Nusse R. Convergence of Wnt, beta-catenin, and cadherin pathways. Science 2004;303:1483-7.

35 Xiao $\mathrm{CY}$, Pan YF, Guo XH, et al. Expression of $\beta$-catenin in rheumatoid arthritis fibroblast-like synoviocytes. Scand J Rheumatol. 2011;40:26-33.

36 Chang SK, Noss EH, Chen $M$, et al. Cadherin-11 regulates fibroblast inflammation. Proc Natl Acad Sci U S A. 2011;2011:8402-7.

37 Klein PS, Melton D a. A molecular mechanism for the effect of lithium on development. Proc Natl Acad Sci U S A. 1996;93:8455-9. 
38 Morikawa T, Kuchiba A, Yamauchi M, et al. Association of CTNNB1 (betacatenin) alterations, body mass index, and physical activity with survival in patients with colorectal cancer. JAMA. 2011;305:1685-94.

39 Blüml S, Bonelli M, Niederreiter B, et al. Essential role of microRNA-155 in the pathogenesis of autoimmune arthritis in mice. Arthritis Rheum. 2011;63:1281-8.

40 Kurowska-Stolarska M, Alivernini S, Ballantine LE, et al. MicroRNA-155 as a proinflammatory regulator in clinical and experimental arthritis. Proc Natl Acad Sci U S A. 2011; 108:11193-8.

41 Nakasa T, Shibuya $H$, Nagata $Y$, et al. The inhibitory effect of microRNA-146a expression on bone destruction in collagen-induced arthritis. Arthritis Rheum. 2011;63:1582-90.

42 Garofalo M, Di Leva G, Romano G, et al. miR-221\&222 regulate TRAIL resistance and enhance tumorigenicity through PTEN and TIMP3 downregulation. Cancer cell 2009;16:498-509.

43 Pundt N, Peters $M$ a, Wunrau C, et al. Susceptibility of rheumatoid arthritis synovial fibroblasts to FasL- and TRAIL-induced apoptosis is cell cycledependent. Arthritis Res Ther. 2009;11:R16.

44 Lefèvre S, Knedla A, Tennie C, et al. Synovial fibroblasts spread rheumatoid arthritis to unaffected joints. Nat Med. 2009;15:1414-20. 


\section{Figures Legends}

Figure 1. The TghuTNF-SF miR sequence-based expression profile.

Sequence-based expression profile of significantly dysregulated miRs in TghuTNF-SFs $(n=2)$ compared to WT SFs $(n=2)$. (A), Top 15 upregulated and downregulated miRs. Fold change (Tg / WT) plotted on the $y$-axis and miR abundance in WT SFs is plotted on the x-axis, providing both a measure of dysregulation and expression and (B), Pearson correlation coefficient-based clustering heat map representation of the expression profile, showing that TghuTNF-SFs express a distinct pathogenic profile compared to WT SFs. (C) Average fold change in miR expression in TghuTNF versus WT littermate SFs (Data from 5-8 independent experiments combined). Levels were determined using TaqMan miR assays. Fold change was calculated using the $\Delta \Delta \mathrm{Ct}$ method. All miR levels were normalised to U6 snRNA. Error bars represent \pm SEM. pvalues calculated using a one-sample t-test. bd: Below detection $(C(t)>35)$. (D), Pearson correlation between miR fold change determined by sequencing and qRT-PCR, showing that the sequence-based expression profile is valid and quantitative $(r=$ 0.9782, $\mathrm{p}<0.0001, \mathrm{n}=10)$.

Figure 2. miRs dysregulated in RA versus OA patient SFs.

Expression differences of miRs in RA $(n=8)$ and OA $(n=8)$ SFs. Levels were determined using TaqMan miR assays. Relative expression differences were calculated using the $\Delta \mathrm{C}(\mathrm{t})$ method. All miR levels were normalised to let-7a miR. Bars represent the mean. p-values were calculated using an unpaired t-test, ns: not significant. 
Figure 3. miR-323-3p overexpression, but to not miR-221/222, enhances WNT pathway activation and decreases the levels of it's predicted target BTRC.

(A), WNT reporter activity in HEK 293T cells transfected for 48 hours with 50nM premiR-323-3p or 50nM pre-miR-221/222 (25nM of each miR-221 and miR-222) or 50nM pre-miR-scr (control). WNT activation was induced by treatment with $10 \mathrm{mM} \mathrm{LiCl}$ for 16h. Luciferase activity was normalised for transfection efficiency by GFP expression and is presented as relative fluorescence units (RFU). Results show that miR-323-3p, but not miR-221/222, enhances Wnt activation. Error bars represent \pm SEM and $n=12$ transfection for each pre-miR.

(B), Expression differences of CSNK1A1, BTRC and GSK3B, negative regulators of $\beta$ catenin, in RA $(n=14)$ and OA $(n=10)$ SFs. Levels were determined by qRT-PCR. Relative expression differences were calculated using the $\Delta \mathrm{C}(\mathrm{t})$ method. All transcript levels were normalised to 18s. (C), Expression differences of BTRC and GSK3B in RASFs $(\mathrm{n}=7$ ) transfected with 50nM pre-miR-323-3p or 50nM pre-miR-scr (control), showing that miR-323-3p overexpression decreases the levels of BTRC. Levels were determined by qRT-PCR. Relative expression differences were calculated using the $\Delta \Delta \mathrm{C}(\mathrm{t})$ method. All transcript levels were normalised to $18 \mathrm{~s}$. Bars represent the mean. (D), Pearson correlation between miR-323-3p and BTRC expression in RASFs $(n=12)$, showing an significant inverse correlation $(r=-0.5336, p=0.037)$. $p$-values were calculated using an unpaired t-test, ns: not significant.

Figure S1. Purity of synovial fibroblasts used for miR-seq determined by FACS analysis. Representative overlaid histograms showing the percentage of cells and intensity of expression of CD90.2 and CD-11b, in SFs isolated from 2xWT and 2xTghuTNF mice 
cultured for three passages. Black lines represent the unstained control samples. Blue lines represent WTSFs and red line TghuTNF SFs. (A) All cell preps were $>85 \%$ positive for CD90.2, showing similar expression intensity. (B) All cell preps were $<1 \%$ positive for the myeloid cell marker CD11b, showing negligible intensity of expression. 


\section{Supplementary Material}

Table S1. Sequence-based miR expression profile of TghuTNF SFs.

\begin{tabular}{|c|c|c|c|c|}
\hline microRNA & $\begin{array}{c}\text { Fold Change } \\
\text { (Tg / WT) }\end{array}$ & p-value & q-value & WT abundance \\
\hline miR-329 & 9.1 & 0.0493 & 0.002164882 & 16 \\
\hline miR-223 & 6.3 & 0.0462 & 0.002164882 & 9 \\
\hline miR-146a & 6.1 & 0.0193 & 0.002154901 & 32462 \\
\hline miR-495 & 5.5 & 0.0232 & 0.002154901 & 15 \\
\hline miR-221 & 4.0 & 0.0385 & 0.002492094 & 3822 \\
\hline miR-122 & 3.9 & 0.0138 & 0.002154901 & 55 \\
\hline miR-215 & 3.8 & 0.0014 & 0.002154901 & 114 \\
\hline miR-222 & 3.5 & 0.0009 & 0.001131415 & 4990 \\
\hline miR-592 & 2.9 & 0.0148 & 0.001131415 & 14 \\
\hline miR-31 & 2.6 & 0.0374 & 0.002154901 & 1228 \\
\hline miR-155 & 2.6 & 0.0239 & 0.002154901 & 3149 \\
\hline miR-129-3p & 2.3 & 0.0017 & 0.002154901 & 112 \\
\hline miR-694 & 2.2 & 0.0298 & 0.006199645 & 29 \\
\hline miR-138 & 2.2 & 0.0425 & 0.003285265 & 122 \\
\hline miR-22 & 2.0 & 0.0392 & 0.002875388 & 93439 \\
\hline miR-667 & 1.8 & 0.0188 & 0.002164882 & 112 \\
\hline miR-344 & 1.8 & 0.0399 & 0.004472551 & 80 \\
\hline
\end{tabular}




\begin{tabular}{|c|c|c|c|c|}
\hline miR-140* & 1.7 & 0.0486 & 0.001131415 & 265858 \\
\hline miR-770-3p & 1.6 & 0.0490 & 0.002154901 & 103 \\
\hline miR-382 & 1.6 & 0.0367 & 0.003371155 & 6208 \\
\hline miR-323-3p & 1.5 & 0.0428 & 0.002164882 & 42 \\
\hline miR-433 & 1.5 & 0.0182 & 0.00350631 & 517 \\
\hline miR-669b & -1.5 & 0.0350 & 0.002408699 & 980 \\
\hline miR-297a & -1.5 & 0.0185 & 0.002154901 & 1226 \\
\hline miR-669f & -1.6 & 0.0213 & 0.002787427 & 77 \\
\hline miR-466f & -1.6 & 0.0314 & 0.00350631 & 955 \\
\hline miR-467f & -1.6 & 0.0378 & 0.005546369 & 918 \\
\hline miR-466d-5p & -1.7 & 0.0068 & 0.002154901 & 73 \\
\hline miR-126-5p & -1.7 & 0.0244 & 0.002605797 & 298 \\
\hline miR-126-3p & -1.7 & 0.0399 & 0.004237086 & 2208 \\
\hline miR-467d & -1.8 & 0.0166 & 0.002408699 & 1226 \\
\hline miR-676 & -2.1 & 0.0097 & 0.002154901 & 9341 \\
\hline miR-466k & -2.1 & 0.0164 & 0.007619994 & 38 \\
\hline miR-466g & -2.1 & 0.0375 & 0.003319105 & 113 \\
\hline miR-676* & -2.1 & 0.0097 & 0.002408699 & 1910 \\
\hline miR-455* & -2.3 & 0.0405 & 0.002164882 & 360 \\
\hline miR-378 & -2.6 & 0.0225 & 0.004985264 & 66737 \\
\hline miR-708 & -2.7 & 0.0130 & 0.002164882 & 313 \\
\hline miR-203 & -2.7 & 0.0271 & 0.003319105 & 1334 \\
\hline miR-378* & -2.7 & 0.0025 & 0.004579373 & 47 \\
\hline
\end{tabular}




\begin{tabular}{|c|c|c|c|c|}
\hline miR-542-3p & -2.9 & 0.0375 & 0.004413499 & 708 \\
\hline miR-450a-5p & -3.0 & 0.0459 & 0.002164882 & 128 \\
\hline miR-708* & -3.0 & 0.0488 & 0.002767633 & 49 \\
\hline miR-322 & -3.5 & 0.0377 & 0.009036641 & 1862 \\
\hline miR-146b* & -4.1 & 0.0252 & 0.002711552 & 120 \\
\hline miR-503* & -4.1 & 0.0267 & 0.003777431 & 211 \\
\hline miR-542-5p & -4.3 & 0.0022 & 0.002154901 & 64 \\
\hline miR-503 & -4.9 & 0.0354 & 0.002164882 & 152 \\
\hline miR-483 & -6.2 & 0.0333 & 0.006199645 & 158 \\
\hline miR-204 & -8.1 & 0.0270 & 0.003782695 & 149 \\
\hline miR-335-3p & -14.2 & 0.0144 & 0.006619368 & 460 \\
\hline miR-335-5p & -19.7 & 0.0495 & 0.003146091 & 490 \\
\hline
\end{tabular}

Significantly dysregulated (Fold Change $\geq 1.5, \mathrm{p}$-value $<0.05, \mathrm{FDR}<5 \%$ ) miRs in TghuTNF SFs $(n=2)$ compared to WT SFs $(n=2)$. miR normalised copy number also provided as a measure of expression. 
Table S2. qRT-PCR measurements of TghuTNF miRNA expression.

\begin{tabular}{ccccc}
\hline miRNA & $\begin{array}{c}\text { No. of } \\
\text { experiments }\end{array}$ & $\begin{array}{c}\text { Fold Change } \\
(\text { Tg / WT })\end{array}$ & Standard Error & P-value \\
\hline miR-146a & 6 & 7.15 & 1.54 & 0.0105 \\
miR-223 & 5 & 6.59 & 1.40 & 0.0163 \\
miR-221 & 6 & 4.39 & 0.77 & 0.0069 \\
miR-222 & 6 & 3.95 & 1.04 & 0.0363 \\
miR-155 & 7 & 2.48 & 0.54 & 0.0337 \\
miR-323-3p & 8 & 1.65 & 0.21 & 0.0186 \\
miR-322 & 5 & $0.60(-1.67)$ & 0.13 & 0.0387 \\
miR-335 & 5 & $0.10(-10.00)$ & 0.04 & $P<0.0001$ \\
miR-138 & 6 & 1.03 & 0.22 & 0.8828 \\
miR-503 & 6 & $0.72(-1.39)$ & 0.24 & 0.2923 \\
miR-122a & 5 & bd & na & na \\
miR-203 & 5 & bd & na & na \\
miR-378 & 5 & bd & na & na \\
\hline
\end{tabular}

Average fold change in miR expression in TghuTNF versus WT littermate SFs. Expression levels were measured using qRT-PCR. Fold changes were calculated using the $\Delta \Delta \mathrm{C}(\mathrm{t})$ method. All miR levels were normalised to U6 snRNA. p-values were calculated using a one sample t-test. bd: Below detection: $C(t)>35$, na: not applicable.

Table S3. qRT-PCR measurements of miRNA expression in RASFs and OASFs.

\begin{tabular}{cccc}
\hline miR & RASF & OASF & p-value* \\
\hline miR-221 & $2.12 \pm 0.6$ & $1 \pm 0.21$ & $\mathbf{0 . 0 4 9}$ \\
miR-222 & $1.72 \pm 0.26$ & $1 \pm 0.18$ & $\mathbf{0 . 0 1 9 2}$ \\
miR-323-3p & $1.78 \pm 0.29$ & $1 \pm 0.2$ & $\mathbf{0 . 0 4 4 4}$ \\
miR-155 & $1.29 \pm 0.1$ & $1 \pm 0.09$ & $\mathbf{0 . 0 4 5}$ \\
miR-146a & $0.4 \pm 0.1$ & $1 \pm 0.37$ & 0.3069 \\
miR-223 & $2.23 \pm 0.85$ & $1 \pm 0.21$ & 0.2288 \\
miR-424 & $1.62 \pm 0.35$ & $1 \pm 0.32$ & 0.1655 \\
miR-335 & $1.07 \pm 0.25$ & $1 \pm 0.2$ & 0.8507 \\
\hline
\end{tabular}

Average miR expression \pm standard error in RASFs and OASFs. Expression levels were measured using qRT-PCR. Expression was calculated using the $\Delta \Delta \mathrm{Ct}$ method. All miR levels were normalised to let-7a. ${ }^{*}$-values were calculated using a two-sided t-test comparing RASF $\Delta \mathrm{C}(\mathrm{t})$ and OASF $\Delta \mathrm{C}(\mathrm{t})$. 
Table S4. Characteristics of the study patients

\begin{tabular}{|c|c|c|}
\hline & $\begin{array}{c}\text { RA patients } \\
(\mathrm{n}=8)\end{array}$ & $\begin{array}{c}\text { OA patients } \\
(n=8)\end{array}$ \\
\hline Age, mean (range), years & $66.3(51-80)$ & $67.8(52-87)$ \\
\hline Sex, no. female/male & $7 / 1$ & $2 / 6$ \\
\hline Disease duration, mean (range), years & $23.9(5-50)$ & NA \\
\hline \multicolumn{3}{|l|}{ Medications, no. taking } \\
\hline NSAIDs & 2 & 3 \\
\hline MTX & 4 & 0 \\
\hline leflunomid & 2 & 0 \\
\hline steroids & 3 & 0 \\
\hline tocilizumab & 2 & 0 \\
\hline No. RF+ (>20 IU)/no.assessed & $6 / 7$ & NA \\
\hline DAS28, mean (range) & $2.1(1.38-2.8)$ & NA \\
\hline
\end{tabular}
influence miR expression levels. 
Figures

Figure 1

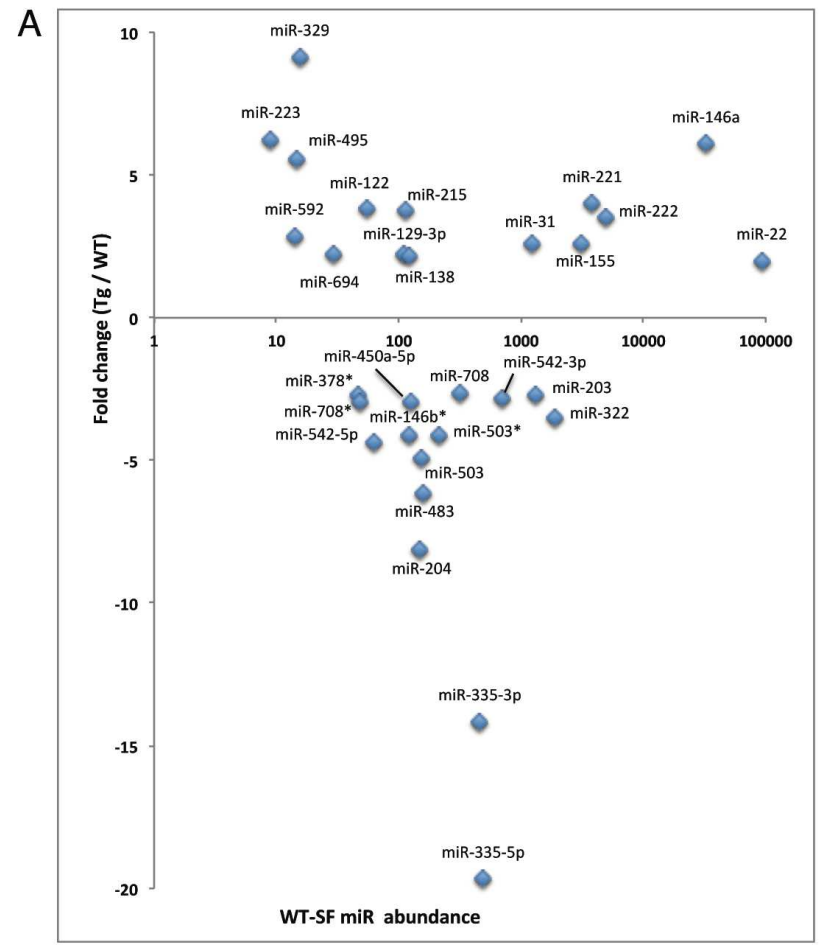

B

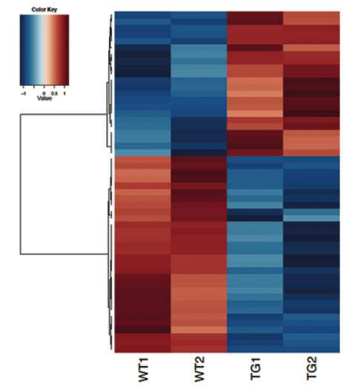

C miR-335-h $p<0.0001$

miR-503- $\square$

miR-322- $-\mathrm{r}=0.0387$

miR-203-bd

miR-378-bd

miR-323-3p- $-1 \mathrm{p}=0.0186$

miR-138-

$\operatorname{miR}-155-\longrightarrow p=0.0337$

$\mathrm{miR}-222$

miR-221 $\mathrm{p}=0.0069$

miR-146a- $\longrightarrow p=0.0105$

miR-223 -

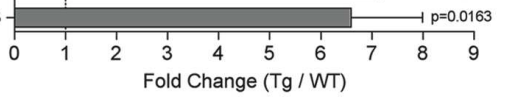

D

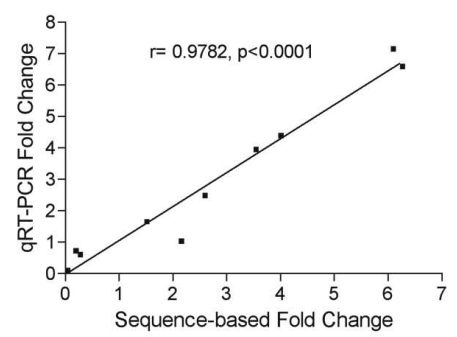

Figure 2
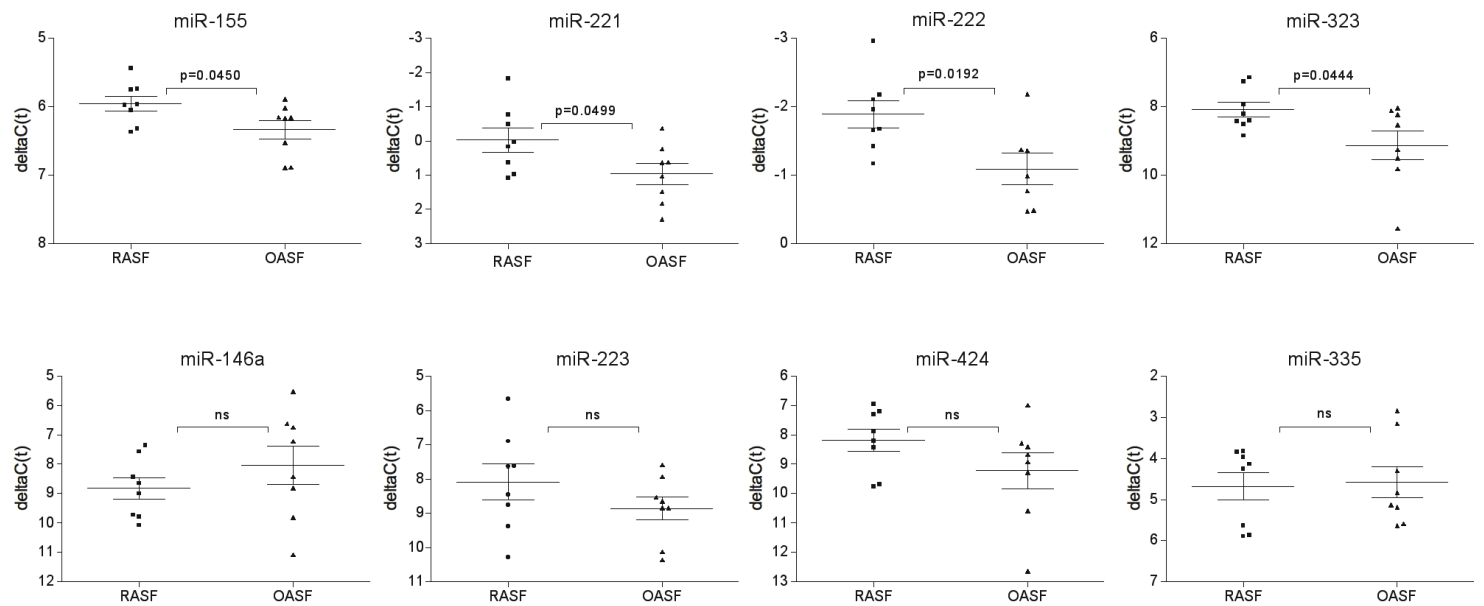
Figure 3

A

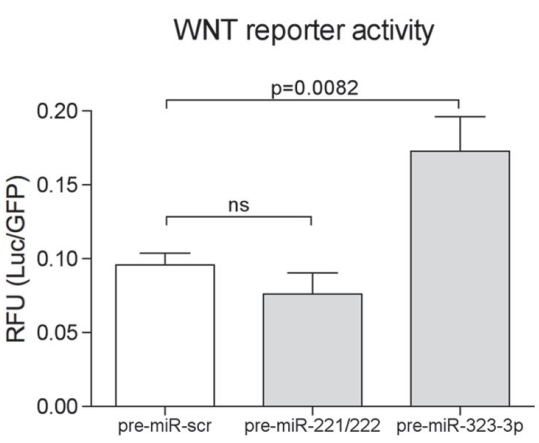

C

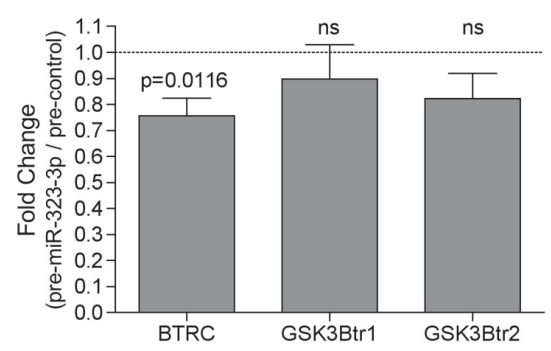

B
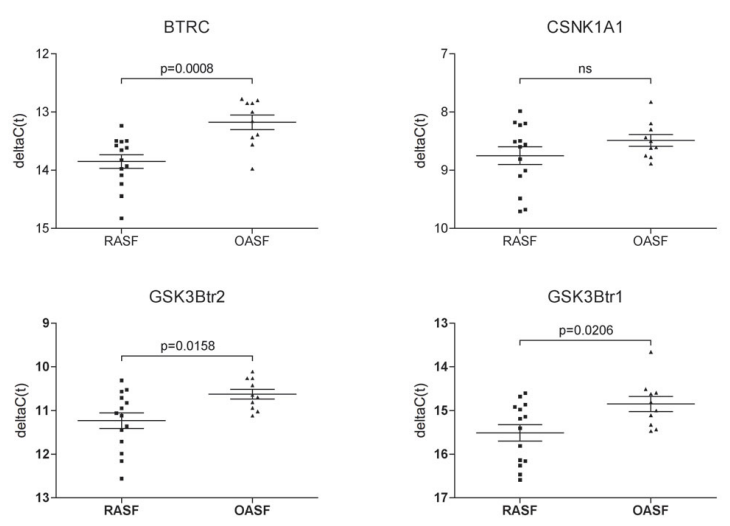

D

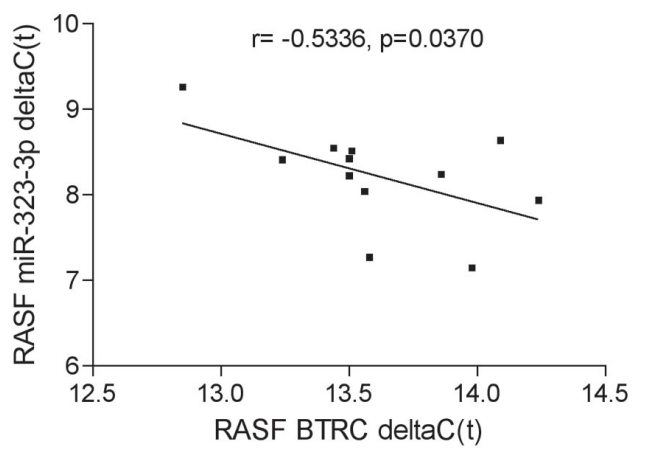

Figure S1

A

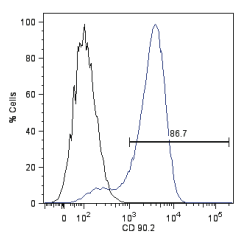

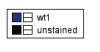

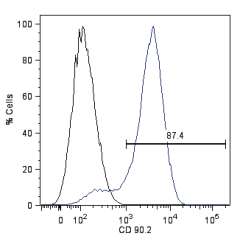

B

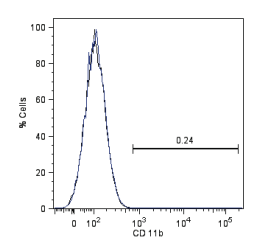

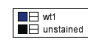

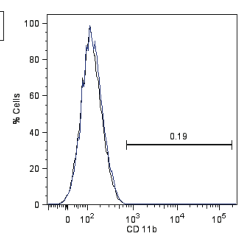

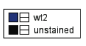

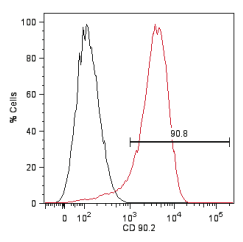

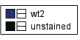

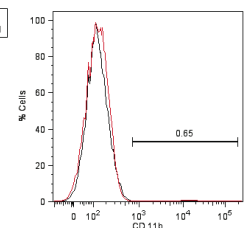

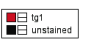

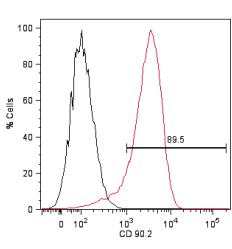

H:

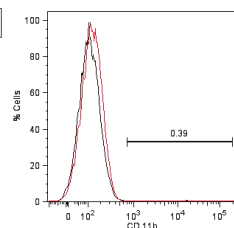

